\title{
Evolução da Nova Doença Infecciosa do Coronavírus (COVID-19) no Brasil até a Semana Epidemiológica 53 de 2020
}

\section{Evolution of the New Infectious Coronavirus Disease (Covid-19) in Brazil Until Epidemiological Week 53 of 2020}

\author{
Francisco Railson Bispo de Barros ${ }^{1}$
}

\section{RESUMO}

Objetivo: Caracterizar o perfil epidemiológico da COVID-19 no Brasil até a Semana Epidemiológica 53 de 2020. Metodologia: Trata-se de um estudo ecológico baseado em dados e documentos preexistentes do Ministério da Saúde brasileiro e órgãos de saúde internacionais, conduzido a partir de uma análise comparativa do Brasil, suas macrorregiões e unidades federativas, com os outros quatro países com maior número de casos e óbitos registrados, tendo sido calculadas as taxas de incidência e de mortalidade. Resultados: Ao final da semana epidemiológica 53, o Brasil registrou 7.716.405 casos, 195.725 óbitos e todos os municípios com registros oficiais da COVID-19. O Brasil estava em terceiro lugar em casos acumulados, atrás de Estados Unidos (19.806.654) e Índia (10.279.788), e em segundo em óbitos, após os Estados Unidos (342.830) e seguido da Índia (149.218). A macrorregião com as maiores taxas foi a Centro-Oeste (53.835,8/1 milhão). O Estado de Roraima apresentou a maior taxa de incidência do país (113.671,8/1 milhão) e o Estado do Rio de Janeiro a maior taxa de mortalidade (1.483,2/1 milhão). Conclusão: O Brasil se encontra em ampla disseminação da COVID-19, demonstrando crescimento e decréscimo regional em fases diferentes, especialmente, em contextos sociossanitários desfavoráveis, o que consequentemente reflete no maciço contágio, adoecimento e óbitos.

\section{DESCRITORES}

Coronavírus. Pandemias. Vigilância em Saúde Pública. Epidemiologia.

\begin{abstract}
Objective: to characterize the epidemiological profile of COVID-19 in Brazil until Epidemiological Week 53 of 2020. Methodology: it is an ecological study based on data and preexisting documents from the Brazilian Ministry of Health and international health agencies, conducted from a comparative analysis of Brazil, its macro-regions, and Federative Units, with the other four countries with the highest number of registered cases and deaths, and calculated incidence and mortality rates. Results: by the end of the epidemiological week 53, Brazil registered 7.716 .405 cases, 195.725 deaths and all municipalities with official records of COVID-19. Brazil was in third place in accumulated cases, behind the United States (19.806.654) and India (10.279.788), and in second in deaths, behind the United States (342.830) and followed by India (149.218). The macro-region with the highest rates was the Midwest (53.835.8/1 million). The state of Roraima had the highest incidence rate in the country (113.671.8/1 million), and the state of Rio de Janeiro had the highest mortality rate (1.483.2/1 million). Conclusion: Brazil is in a widespread dissemination of COVID-19, showing regional growth and decrease in different phases, especially in unfavorable social and health contexts, which consequently reflects in the massive contagion, illness and deaths.
\end{abstract}

\section{DESCRIPTORS}

Coronavirus. Pandemics. Public Health Surveillance. Epidemiology.

\footnotetext{
${ }^{1}$ Programa de Pós-Graduação em Medicina Tropical da Universidade do Estado do Amazonas (UEA), Manaus, Amazonas, Brasil.
} 
A té o início do mês de dezembro de 2019, seis cepas diferentes de coronavírus eram conhecidas por infectar seres humanos, a saber: Coronavírus Humano 229E (HCoV229E), Coronavírus Humano (HCoV-OC43), Síndrome Respiratória Aguda Grave Coronavírus (SARS-CoV), agente etiológico que foi responsável por um surto de doença respiratória na China durante os anos de 2002 e 2003, HCoV-NL63 (NL63), HCoVHKU1 (HKU1) e Síndrome Respiratória do Oriente Médio Coronavírus (MERSCoV), patógeno que desencadeou um surto contínuo de doença respiratória grave centrado no Oriente Médio desde 2012².

No final do mês de dezembro de 2019 , foram relatados nove casos de pacientes internados em três diferentes hospitais da Cidade de Wuhan, na província de Hubei, China, com diagnóstico de pneumonia viral de causa não identificada. Oito dos nove pacientes foram encontrados geograficamente associados ao mercado de frutos do mar de Huanan em Wuhan, apontando o mercado como a possível origem de infecção ${ }^{2,3}$.

A partir da análise dos sequenciamentos de genoma de amostras de fluido de lavagem broncoalveolar e isolados de cultura dos nove pacientes, foi possível descrever e estruturar um sétimo coronavírus que infecta seres humanos, provisoriamente intitulado em 2019 novo coronavírus (nCoV-2019), que mais tarde foi denominado Síndrome Respiratória Aguda Grave-Coronavírus 2 (SARS-CoV-2) e agente etiológico da doença do coronavírus 2019 (COVID-19), destacando o reservatório oculto do vírus em animais selvagens e seu potencial de ocasionalmente se espalhar para as populações humanas de uma forma sem precedentes ${ }^{4}$.

A Organização Mundial de Saúde (OMS), no dia 9 de janeiro de 2020, afirmou que um novo coronavírus se encontrava em circulação. O primeiro caso de relevância epidemiológica territorial foi notificado em solo japonês no dia 16 de janeiro e cinco dias depois, nos Estados Unidos da América, foi confirmado o primeiro caso importado. No dia 30 de janeiro de 2020, a OMS declarou o surto do SARS-CoV-2 como uma Emergência de Saúde Pública de Importância Internacional (ESPII), uma vez que já existiam confirmações de casos importados em 19 países, com transmissão entre humanos na China, Alemanha, Japão, Vietnã e Estados Unidos da América ${ }^{5}$.

Em 11 de março de 2020, a COVID-19 foi classificada pela OMS como uma pandemia. No dia 2 de janeiro de 2021, final

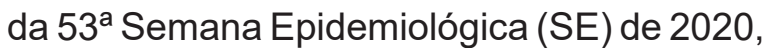
a OMS tinha confirmado 84.586.904 casos de COVID-19 no mundo. O país com maior número de casos acumulados (20.426.184) era os Estados Unidos da América, acompanhado da Índia (10.323.965), Brasil (7.716.405), Federação Russa (3.179.898) e França $(2.700 .480)^{6,7}$.

No Brasil, a resposta do Ministério da Saúde (MS) frente à doença emergente foi o acionamento do Centro de Operações de Emergência (COE), vinculado à Secretaria de Vigilância em Saúde (SVS/MS), para articular, planejar e ordenar as implementações com os setores envolvidos e acompanhar o contexto epidemiológico. Assim, no dia 26 de fevereiro de 2020, o MS recebeu a primeira 
notificação de um caso confirmado do novo coronavírus na Cidade de São Paulo, em 17 de março o primeiro óbito, também de São Paulo, e no dia 20 de março fora reconhecida a transmissão comunitária em todo o território nacional ${ }^{8}$

O espectro clínico da infecção pelo SARS-CoV-2 é amplo. O vírus apresenta tropismo pelos tecidos superiores e inferiores do aparelho respiratório, característica que evidencia as mucosas nasal, bucal e ducto lacrimal como as predominantes vias de entrada no organismo humano ${ }^{2-4}$. Quando um indivíduo infectado tosse ou espirra, dispersa saliva e aerossóis contendo o vírus, o que consequentemente torna o meio no qual as partículas são inseridas potenciais fontes de transmissão, uma vez que o vírus pode permanecer viável e infeccioso em aerossóis por horas e em superfícies por até dias ${ }^{9,10}$.

Destaca-se que a consolidação dos dados preexistentes sobre os casos e óbitos por COVID-19, coletados e disponibilizados pelas Secretarias Estaduais de Saúde, vem sendo realizada desde o primeiro registro da doença no Brasil pelo MS. Isso permite o conhecimento da dinâmica da doença no país e, consequentemente, o planejamento e a implementação de políticas para desacelerar o crescimento do número de casos e óbitos ${ }^{11}$.

Em vista disso, este estudo objetivou descrever a evolução da COVID-19 no Brasil até a semana epidemiológica (SE) 53. Tal evolução foi comparada entre os cinco países com maior número de casos da COVID-19 notificados até esta semana, bem como entre as Unidades da Federação (UFs).

\section{METODOLOGIA}

Este estudo caracteriza-se como ecológico descritivo, cujas unidades de análise foram o Brasil, suas macrorregiões e UFs, assim como os demais quatro países que apresentaram maior número de casos confirmados (Estados Unidos da América, Índia, Rússia, França) e quatro países que apresentaram maiores números de óbitos confirmados (Estados Unidos da América, Índia, México, Itália) de COVID-19 até 2 de janeiro de 2021, o último dia da SE 53.

A situação epidemiológica foi contextualizada a partir do dia 26 de fevereiro, data da confirmação do primeiro caso de COVID-19 no Brasil e até 2 de janeiro de 2020, último dia da SE 53 e data de extração dos dados. Foram utilizados os dados de casos e óbitos confirmados pela doença, por local de residência e agregados por país, macrorregião geográfica e UFs do país, disponibilizados pelo Painel COVID-19 do MS e, para comparação com a situação mundial, foram considerados os informes epidemiológicos disponibilizados pela OMS e pela Johns Hopkins University 7,8,13.

Sobre o número de casos confirmados por COVID-19 no Brasil, é importante destacar que o MS adotou diferentes definições operacionais durante a pandemia, sendo que no dia 5 de agosto de 2020 divulgou a atualização do Guia de Vigilância Epidemiológica Emergência de Saúde Pública de Importância Nacional pela Doença pelo Coronavírus 2019, no qual definiu casos confirmados por critério clínico, critério clínico-epidemiológico e critério clínico-imagem ${ }^{14}$. 
Foram elaborados gráficos de casos e óbitos novos e acumulados por SE para os cinco países e cinco UFs do Brasil com maior número de casos até o dia 2 de janeiro de 2021, a partir da confirmação do $50^{\circ}$ caso ou óbito, com o intuito de descrever em que fase da epidemia o Brasil e suas UFs estão e como estão se comportando as curvas epidêmicas de cada localidade. Para o levantamento dos fatos históricos, foram consultados o sítio eletrônico da OMS, a literatura especializada e documentos oficiais disponibilizados pelo MS brasileiro ${ }^{7-8,13,15,16}$

Por fim, foram calculadas as taxas de incidência e de mortalidade para os países e para o Brasil, por macrorregiões e UFs, obtidas dividindo-se o número de casos e óbitos, respectivamente, pela população residente e multiplicando-se por 1 milhão. Esse fator de multiplicação foi utilizado para permitir comparações nacionais e internacionais. As estimativas populacionais utilizadas como denominadores do mundo foram produzidas pelo Banco Mundial ${ }^{17} \mathrm{e}$ as do Brasil foram produzidas pelo Instituto Brasileiro de Geografia e Estatística (IBGE) para o Tribunal de Contas da União (TCU) e são referentes ao ano de $2019^{18}$. O software Microsoft Excel e R 3.5.3 foram utilizados para tratamento, análise de dados e criação de gráficos.

Conforme as orientações da Resolução $n^{\circ} 466 / 2012$, do Conselho Nacional de Saúde, a aprovação por um Comitê de Ética em Pesquisa com Seres Humanos não foi necessária devido ao uso de dados não identificados e publicamente disponíveis ${ }^{19}$.

\section{RESULTADOS}

O primeiro caso da COVID-19 foi identificado em 1 de dezembro de 2019, mas só foi reportado em 31 de dezembro do mesmo ano e, até o dia 2 de janeiro de 2021, final da SE 53 de 2020, foram registrados 82.940.272 casos em 223 países e 1.825.110 óbitos confirmados em 204 destes pela doença. Em 2 de janeiro de 2021, os Estados Unidos da América apresentavam o maior número de casos $(19.806 .654$; 59.838,3 por 1 milhão de hab.), acompanhado da Índia (10.279.788; 7.449,0 por 1 milhão de hab.), Brasil (7.716.405; 36.719,0 por 1 milhão de hab.), Federação Russa (3.212.637; 22.014,2 por 1 milhão de hab.) e França (2.595.661; 39.765,9 por 1 milhão de hab.). Naquela data, o Brasil ocupava a $3^{a}$. posição em números absolutos de casos confirmados e a $2^{\mathrm{a}}$. posição segundo óbitos confirmados. Os maiores números de óbitos foram encontrados nos Estados Unidos da América (342.830; 1.035,7 óbitos por 1 milhão de hab.), seguido do Brasil (195.725; 931,3 óbitos por 1 milhão de hab.), Índia (149.218; 108,1 óbitos por 1 milhão de hab.), México (125.807; 975,7 óbitos por 1 milhão de hab.) e a Itália (74.621; 1.234,1 óbitos por 1 milhão de hab.) (Figuras $1 \mathrm{~A} \mathrm{e} \mathrm{1B}$ ).

Comparando-se a evolução do número de casos novos registrados por COVID-19 por SE dos cincos países mais afetados pela doença após o $50^{\circ}$ caso, evidenciou-se que cada país se encontrava em uma fase específica da pandemia. Brasil (dia 280) registrou a maior soma de casos novos na SE 51 (70.570 casos) e na SE 53 apresentou uma diminuição no número 
de casos novos. Em relação à evolução do número de óbitos novos por dia após o $50^{\circ}$ óbito, observou-se que o Brasil (dia 125) registrou o maior número na SE 31. Vale ressaltar que as curvas de novos casos e óbitos do Brasil se portam de forma distinta, demonstrando crescimento e decréscimo em fases diferentes quando contrastadas com os demais países.

O MS brasileiro recebeu a primeira notificação de um caso confirmado pelo Estado de São Paulo no dia 26 de fevereiro. O paciente era um brasileiro do sexo masculino, 61 anos, que viajou à Itália, dando entrada hospitalar um dia antes. No dia 22 de março, existem registros de casos da doença nos 26 estados e no Distrito Federal. Quanto ao registro de óbitos, o Estado de São Paulo foi o primeiro a notificar no dia 17 de março, onde o paciente tinha realizado viagem ao exterior e apresentava idade avançada como fator de risco associado à mortalidade. Em 16 de março, as Capitais do Rio de Janeiro e São Paulo registram transmissão comunitária, no dia 20 do mesmo mês, o MS declarou transmissão comunitária em todo o solo brasileiro, fazendo com que o país entrasse em nova fase do Plano de Contingência

Figura 1. Evolução do número de novos casos, segundo os cinco países com maior número de casos e óbitos até o dia 2 de janeiro de 2021

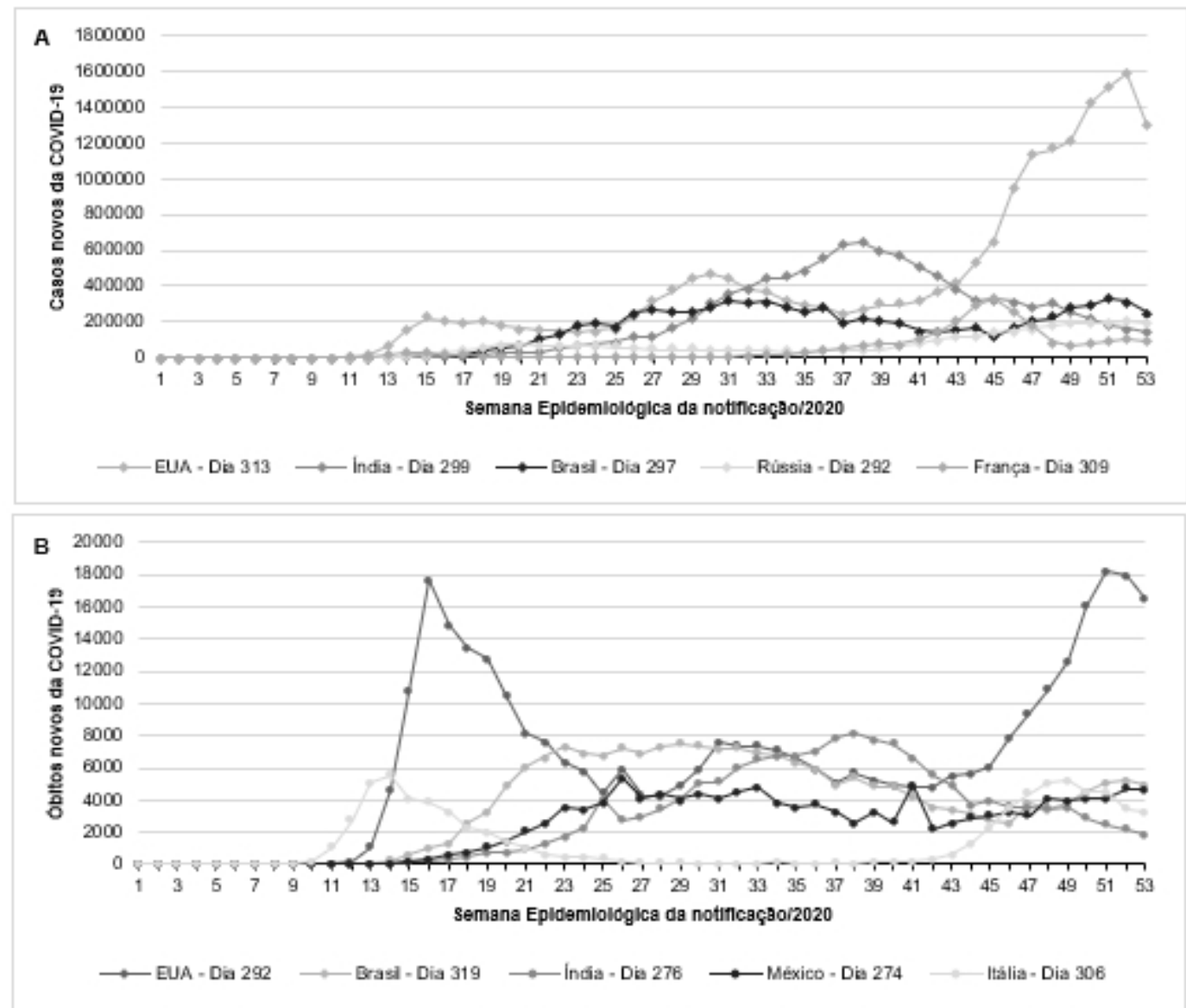

Fonte: Our Word in Data (2021). (A) e óbitos (B) confirmados de COVID-19 por Semana Epidemiológica. 
Figura 2. Distribuição semanal dos casos a partir do $1^{\circ}$ registro, respectivamente, entre as macrorregiões do Brasil até o dia 2 de janeiro de 2021

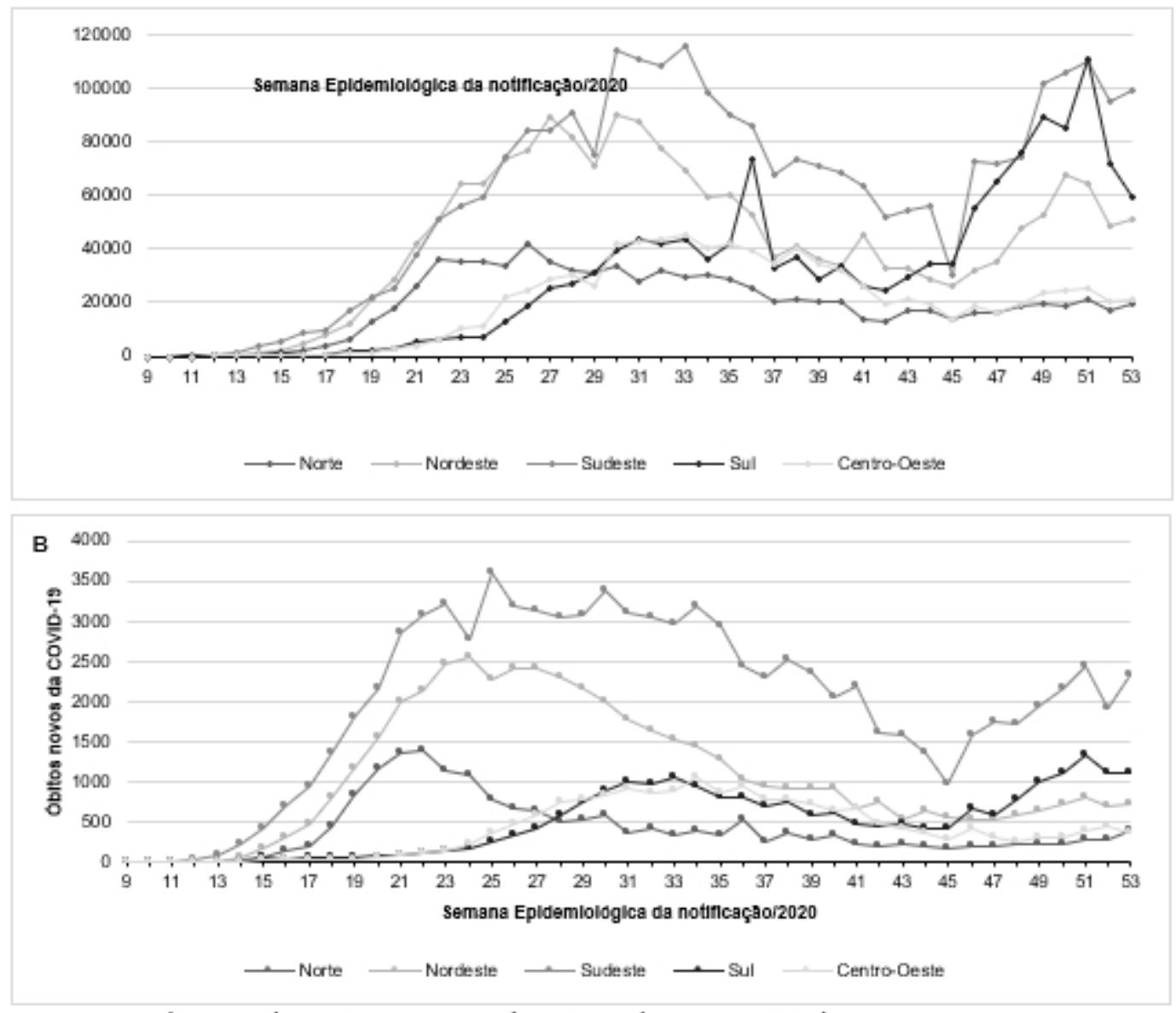

Fonte: Ministério da Saúde; Secretaria de Vigilância em Saúde (2021). (A) e óbitos (B) novos por COVID-19

Nacional. Desde então, o Brasil está entre as nações com maior número de casos e óbitos acumulados no mundo.

A Figura 2 reproduz o transcorrer das SE de 2020, na qual se pode observar heterogeneidade entre as curvas de casos e óbitos novos da COVID-19 nas macrorregiões do Brasil. As macrorregiões Sudeste, Nordeste e Norte mostram crescimento do número de casos e óbitos anteriormente a SE 16, ao mesmo tempo em que nas macrorregiões Sul e Centro-Oeste tal fenômeno foi evidenciado somente após a SE 20. Ao longo das SE 9 a 53, foram contabilizados 7.716 .405 casos e 195.725 óbitos no país, onde a macrorregião Sudeste apresentava o maior número de casos (2.703.086) e óbitos (89.552), seguida pelo Nordeste (1.904.954 casos; 47.926 óbitos), Sul (1.369.059 casos; 22.229 óbitos), CentroOeste (877.367 casos; 17.908 óbitos) e Norte (861.939 casos; 18.110 óbitos).

Conforme pode ser observado na Tabela 1, até o final da SE 53, o Brasil registrou uma taxa de incidência de 36.719,0/1 milhão de hab., enquanto a taxa de mortalidade 
Tabela 1. Distribuição dos registros de casos e óbitos novos por covid-19 no Brasil, segundo região e Unidade da Federação (UF), até o dia 2 de janeiro de 2021. Brasil (2020-2021)

\begin{tabular}{|c|c|c|c|c|c|c|c|}
\hline Repiāo/UF & $\begin{array}{l}\text { População } \\
\text { TCu/2019 }\end{array}$ & $\begin{array}{c}\mathrm{N}^{\circ} \text { de dias } \\
\text { após o } 1^{\circ} \\
\text { casso }\end{array}$ & Casos N & $\begin{array}{l}\text { Taxa de } \\
\text { incidencia' }\end{array}$ & $\begin{array}{l}\mathrm{N}^{\circ} \text { de dias } \\
\text { após o } 1^{\circ} \\
\text { dbito }\end{array}$ & Obiitos N & $\begin{array}{c}\text { Taxa de } \\
\text { mortalidade }\end{array}$ \\
\hline Norte & 18.430 .980 & 294 & 861.939 & 46.765 .7 & 284 & 18. 110 & 982,5 \\
\hline Acre & 881.935 & 290 & 41.941 & $47.555,6$ & 271 & 798 & 904,8 \\
\hline Amazonas & 4.144 .597 & 294 & 201.867 & $48.706,0$ & 284 & 5.325 & $1.284,8$ \\
\hline Amapś & 845.731 & 289 & 68.441 & $80.925,2$ & 274 & 927 & $1.096,0$ \\
\hline Pará & 8.602 .865 & 290 & 293.807 & $3.4152,2$ & 277 & 7.209 & 837,9 \\
\hline Roraima & 1.777 .225 & 289 & 96.433 & 54260,4 & 278 & 1.825 & $1.026,8$ \\
\hline Rondônia & 605.761 & 277 & 68.858 & $113.671,8$ & 274 & 787 & $1.299,1$ \\
\hline Tocantins & 1.572 .866 & 290 & 90.592 & $57.596,7$ & 262 & 1.239 & 787,7 \\
\hline Nordeste & 57.071 .654 & 303 & 1.904 .954 & $33.378,2$ & 284 & 47.926 & 839,7 \\
\hline Alagoas & 3.337 .357 & 301 & 105.361 & $31.570,1$ & 278 & 2.502 & 749,6 \\
\hline Bahia & 14.873 .064 & 303 & 495.286 & $33.300,8$ & 280 & 9.187 & 617,6 \\
\hline Ceará & 9.132 .078 & 292 & 336.574 & $36.856,2$ & 283 & 10.015 & $1.096,6$ \\
\hline Maranhăิo & 7.075 .181 & 288 & 200.976 & $28.405,7$ & 279 & 4.513 & 637,8 \\
\hline Paraiba & 4.018 .127 & 290 & 167.615 & $41.714,7$ & 277 & 3.692 & 918,8 \\
\hline Pernambuco & 9.557 .071 & 297 & 223.325 & $23.367,5$ & 284 & 9.674 & $1.012,2$ \\
\hline Piaui & 3.273 .227 & 289 & 143.210 & $43.751,9$ & 281 & 2.848 & 914,9 \\
\hline $\begin{array}{l}\text { Rio Grande do } \\
\text { Norte }\end{array}$ & 3.506 .853 & 296 & 118.691 & $33.845,4$ & 280 & 2.995 & 854,0 \\
\hline Sergipe & 2.298 .696 & 293 & 113.916 & $49.556,7$ & 276 & 2.500 & $1.087,5$ \\
\hline Sudeste & 88.371 .433 & 312 & 2.703 .086 & $30.587,7$ & 292 & 89.552 & $1.013,3$ \\
\hline Espírito Sanso & 4.018 .650 & 303 & 250.227 & $62.266,4$ & 276 & 5.113 & $1.272,3$ \\
\hline Minas Gerais & 21.168 .791 & 301 & 549.302 & $25.948,6$ & 279 & 12.023 & 567,9 \\
\hline Rio de Janeiro & 17.264 .943 & 304 & 435.604 & $25.230,5$ & 290 & 25.608 & $1.483,2$ \\
\hline Săo Paulo & 45.919 .049 & 312 & 1.467 .953 & $31.968,2$ & 292 & 46.808 & $1.019,3$ \\
\hline Sul & 29.975 .984 & 299 & 1.369 .059 & $45.671,8$ & 284 & 22.229 & 741,5 \\
\hline Parană & 11.433 .957 & 297 & 419.615 & $36.699,0$ & 282 & 8.001 & 699,7 \\
\hline $\begin{array}{l}\text { Rio Grande do } \\
\text { Sul }\end{array}$ & 11.377 .239 & 299 & 452.920 & $39.809,3$ & 284 & 8.934 & 785,2 \\
\hline Santa Catarina & 7.164 .788 & 296 & 496.524 & $69.300,5$ & 283 & 5.294 & 738,8 \\
\hline Centro-Oeste & 16.297 .074 & 302 & 877.367 & $53.835,8$ & 283 & 17.908 & $1.098,8$ \\
\hline Distrito Federal & 3.015 .268 & 302 & 252.874 & $83.864,5$ & 280 & 4.268 & $1.415,4$ \\
\hline Goiás & 7.018 .354 & 296 & 309.194 & $44.055,0$ & 283 & 6.805 & 969,6 \\
\hline $\begin{array}{l}\text { Mato Grosso } \\
\text { do Sul }\end{array}$ & 2.778 .986 & 293 & 135.361 & $48.708,7$ & 278 & 2.360 & 849,2 \\
\hline Mato Grosso & 3.484 .466 & 289 & 179.939 & $51.640,0$ & 275 & 4.475 & $1.284,2$ \\
\hline Brasil & 210.147 .125 & 26,02 & 7.716 .405 & $36.719,0$ & 292 & 195.725 & 931,3 \\
\hline
\end{tabular}

foi de 931,3/1 milhão de hab. Entre as macrorregiões, a Centro-Oeste apresentou a maior taxa de incidência (53.835,8/1 milhão de hab.) e mortalidade (1.098,8/1 milhão de hab.) do país. Ainda que o Estado de São Paulo tenha o maior número de casos e óbitos acumulados entre as UFs, Roraima evidenciou a maior incidência, 113.671,8/1 milhão de hab., e o Rio de Janeiro a maior mortalidade, 1.483,2/1 milhão de hab., ambas superando até mesmo a regional e a nacional. Todos os 5.570 municípios brasileiros apresentam registros oficiais da COVID-19, sendo o município de Cedro do Abaeté, Minas Gerais, o último a notificar casos da doença.

Analisando a evolução do número de casos novos registrados por COVID-19 por SE das cinco UFs brasileiras mais afetadas pela doença após o $50^{\circ}$ caso, identificou-se que São Paulo (dia 296) apresentou crescimento do número de casos antes da SE 14, enquanto Minas Gerais (dia 287), Santa Catarina (dia 289), Bahia (dia 286) e Rio Grande do Sul 
Figura 3. Distribuição semanal dos casos a partir do $50^{\circ}$ registro, respectivamente, entre as cinco Unidades Federativas com o maior número de casos e óbitos registrados até o dia 2 de janeiro de 2021

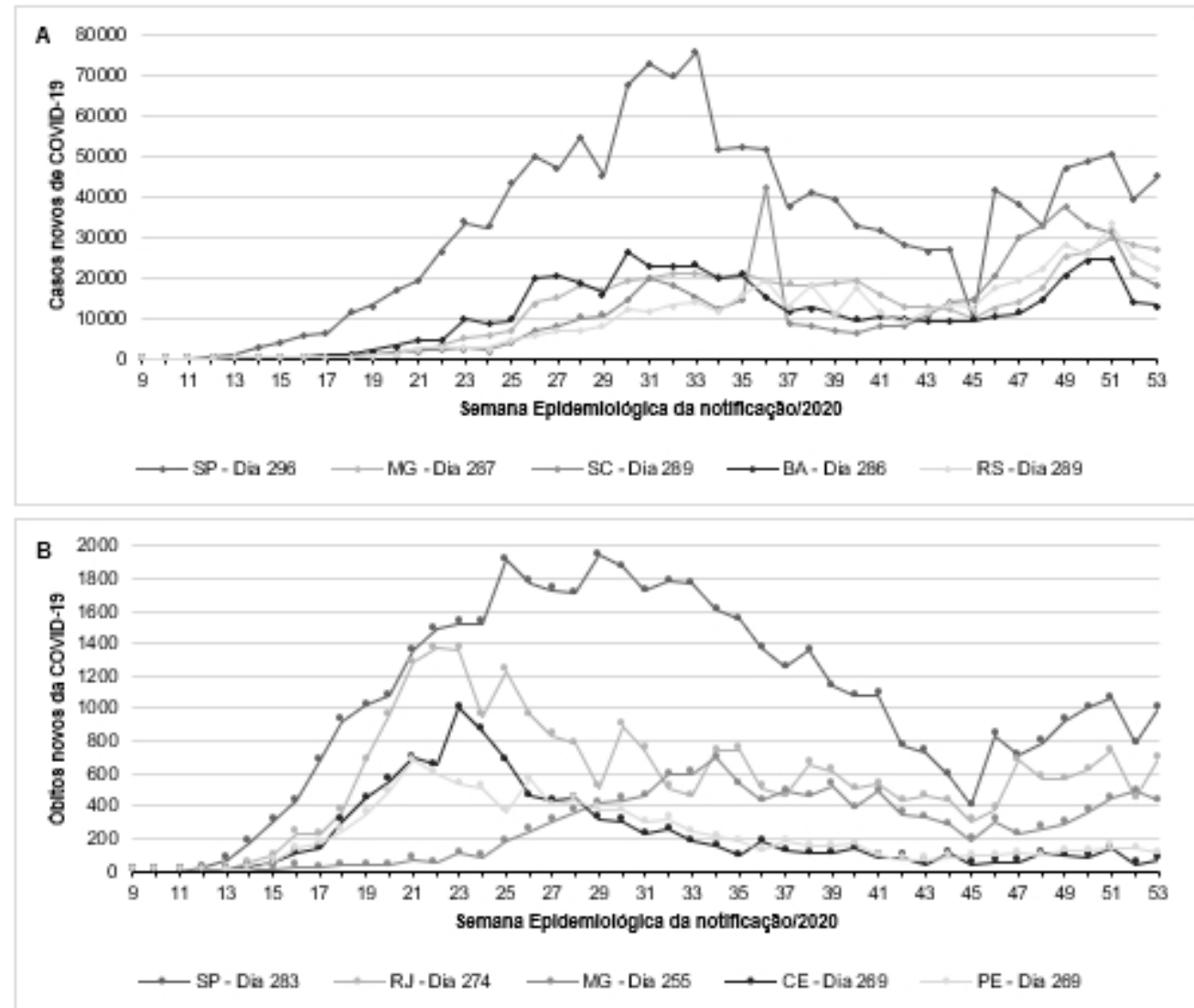

Fonte: Ministério da Saúde; Secretaria de Vigilância em Saúde (2021). (A) e óbitos (B) novos por COVID-19

(dia 289) apresentaram crescimento apenas depois da SE 19. Ao final da SE 53, Minas Gerais e Santa Catarina encontravam-se em dinâmica de redução, Bahia em estabilização, São Paulo e Rio Grande do Sul em incremento (Figura 4A). Quanto à evolução do número de óbitos novos após o $50^{\circ}$ óbito, São Paulo (dia 283), Rio de Janeiro (dia 274), Minas Gerais (dia 255) e Ceará (dia 269) apresentaram crescimento antes da SE 16, ao passo que Pernambuco (dia 269) apresentou crescimento após a SE 20. Ao final da SE 53, Minas Gerais e Pernambuco encontravam-se em dinâmica de redução, São Paulo, Rio de Janeiro e Ceará em incremento (Figura 4b).

\section{DISCUSSÃO}

A COVID-19 tem provocado um crescente número de casos e óbitos no mundo, visto que se configura em uma pandemia de evolução rápida e diária, o 
que consequentemente dificulta quaisquer análises dos dados disponíveis. No entanto, os estudos envolvendo levantamento epidemiológico têm papel expressivo na compreensão sobre o funcionamento, condicionantes e determinantes desta nova doença e sobre a dinâmica na população, de forma geral ou específica, além de determinar o impacto das ações e insumos propostos e desenvolvidos para intervir no seu curso ${ }^{20}$.

Sabe-se que o primeiro registro da COVID-19 ocorreu na China, mas o incremento dos casos e óbitos seguiu dinâmicas diferentes entre os continentes e países com os maiores números acumulados. Ao se analisar os relatórios diários da OMS, a Índia confirmou o primeiro caso da doença no dia 30 de janeiro de 2020, 60 dias depois da China, e na SE 37 tornou-se o segundo maior em número de casos e o terceiro em óbitos. A Rússia confirmou seus primeiros casos um dia depois da Índia, senso o quarto maior em número de casos e o oitavo em óbitos. No continente europeu, a França foi o primeiro país a confirmar casos da doença e vem apresentando inconstâncias entre o crescimento e o decréscimo, mas segue ocupando o quinto lugar no número de casos e o sétimo em óbitos ${ }^{5,8}$.

Os Estados Unidos foram o primeiro país das Américas a confirmar casos importados da COVID-19 e o Brasil o primeiro da América do Sul. Durante a SE 22, a região das américas ultrapassou a europeia em termos de número diário de infecções. Na SE 25, Estados Unidos e Brasil alcançaram a casa do milhão de infectados e se destacaram entre os três países com o maior número de casos e óbitos notificados, fazendo com que as Américas se tornassem o epicentro da pandemia, acumulando mais da metade dos registros ${ }^{5,8,13}$.

Em específico, até o dia 2 de janeiro de 2021, final da SE 53 de 2020, o Brasil ocupava a terceira posição em relação ao número de casos acumulados e o segundo maior número de óbitos registrados, alterando seu perfil epidemiológico que há décadas encontrava-se estabilizado ${ }^{8,16}$. Entretanto, o acompanhamento e registros de casos confirmados da COVID-19 no Brasil se mostram deficitários e com grande proporção de subnotificações, além dos casos assintomáticos e oligossintomáticos, o que coloca o país em uma real situação nebulosa ${ }^{21}$.

As curvas dos cinco países com os maiores quantitativos de casos e óbitos acumulados a partir do $50^{\circ}$ registro, respectivamente, evidenciaram que o Brasil estava em uma fase pandêmica pregressa quando comparado aos outros países até a SE 20 e um crescimento médio de mais de $65 \%$ ao mês até a SE 25,8,8,13,15. Acreditase que a alta velocidade de disseminação da doença, grande desigualdade social entre os países, retardo na deliberação de quarentena, isolamento social e testagem em massa da população, assim como o déficit de leitos, insumos e profissionais de saúde, foram fatores contribuintes para o aumento nas taxas de incidência e mortalidade ${ }^{22,23}$.

Faz-se necessário destacar a inviabilidade de maiores comparações na velocidade de contágio da doença, tanto entre os países quanto entre as regiões e cidades brasileiras, dada a variedade de fatores intrínsecos de cada espaço 
geográfico, a saber: extensão territorial, efetividade de políticas públicas, aspectos culturais e comportamentais, condições de saúde, saneamento e higiene, adensamento e distribuição espacial da população, timing do início da epidemia, contexto político, dentre outros ${ }^{24-26}$.

Neste contexto, o Brasil é o quinto maior país do mundo em área territorial e o sexto mais populoso, características que influenciam diretamente nas diferentes perspectivas de dispersão da doença entre os estados, assim como da alta distribuição dos óbitos. Logo, tanto a variação no tempo provável de incremento dos casos como a falta de contiguidade geográfica, levaram às epidemias assíncronas em diferentes estados brasileiros, destacando-se São Paulo e Rio de Janeiro como fontes internacionais de propagação local inicial, antes das viagens nacionais transportarem o vírus para o resto do país ${ }^{21,27}$.

No final da SE 53, a Macrorregião Sudeste apresentava o maior número de casos e óbitos, e a Centro-Oeste as maiores taxas de incidência e mortalidade. A doença se difundiu pelas regiões brasileiras a partir das principais metrópoles, especificamente São Paulo, Rio de Janeiro, Vitória, Brasília, Salvador, Recife, Fortaleza, Curitiba, Florianópolis e Porto Alegre por via terrestre, Belém e Manaus por via fluvial. Nesta perspectiva, foi identificado que a distribuição dos casos e óbitos entre os cinco estados analisados foi altamente heterogênea, com o Estado de São Paulo e a Região Norte do Brasil apresentando uma velocidade de difusão mais intensa, o que contribuiu para a maior cobertura dos municípios interioranos em menos tempo ${ }^{28}$.
Dessa forma, apesar de o Estado de São Paulo concentrar o maior número de casos acumulados, Roraima foi quem apresentou a maior taxa de incidência e o Rio de Janeiro a maior taxa de mortalidade, ambos superando tanto a média regional quanto a nacional. No entanto, o que se observou durante o mês de dezembro foi o retorno do Estado do Amazonas, especificamente da Capital Manaus, na mídia do Brasil e do mundo devido ao aumento abrupto no número de casos, internações hospitalares e óbitos e, por consequência, o colapso do sistema de saúde público e privado, assim como do sistema funerário ${ }^{29}$.

Este fenômeno pode ser imputado a susceptibilidades comparativas associadas de diferentes grupos de idade, estrutura espacial dos centros urbanos e, principalmente, do nível de base de vários comportamentos individuais (distanciamento social, higienização das mãos e uso de máscaras, são exemplos) associado à redução da transmissão da doença ${ }^{21,27}$. Da mesma forma, superestimação da taxa de ataque do SARS-CoV-2 durante a primeira onda, diminuição da imunidade contra a infecção a partir de dezembro, detecção de novas linhagens do SARS-CoV-2 que escapam da imunidade outrora gerada à infecção e maior poder de transmissibilidade das mesmas, são teorias levantadas para explicar, mas não mutuamente, o ressurgimento da COVID-19 em Manaus ${ }^{29}$.

É neste contexto que autoridades sanitárias e profissionais de saúde têm encontrado obstáculos em elucidar a população brasileira, principalmente das Regiões Norte e Nordeste, quanto ao grau 
de importância das recomendações de entidades e órgãos de saúde internacionais e nacionais no controle da pandemia, a qual é fenomenologicamente envolta de crenças pessoais, cosmogonia massivamente influenciada por fatores históricos, culturais e sociais, que fundamentarão a percepção e escolhas dos indivíduos. Somam-se a isso, as aglomerações irresponsáveis e a propagação de um vírus cibernético denominado fake news, que tende a desacreditar as pesquisas científicas e o cenário ao qual o mundo está imerso ${ }^{30}$.

\section{CONCLUSÃO}

Por meio dos resultados e análises apresentados neste estudo, os quais sofrem alterações diárias, pode-se observar que o incremento do número de casos e óbitos da COVID-19 pelo território brasileiro, apresentou bastante heterogeneidade da SE 9 até o final da SE 53 de 2020, concentrando as maiores taxas de incidência e mortalidade nas regiões consideradas de elevada densidade de relações humanas. 0
Brasil, considerado um país de dimensões continentes, também apresenta um contexto multifacetado de fatores que está entrelaçado ao crescimento dos casos e óbitos da doença, incluindo não somente as características biopsicossociais da população, mas também a vulnerabilidade socioeconômica a qual se encontram expressivos grupos de pessoas, o que reflete na baixa adesão as recomendações de controle da pandemia.

Atualmente, o mundo volta seu olhar para vacinas que estão sendo desenvolvidas e administradas na população, ainda que de forma lenta e heterogênea entre os países. Este fato pode ser atribuído à disseminação de notícias falsas e a politização da pandemia, o que tem trazido resultados negativos e sombrios ao Brasil. Dessa forma, recai nos ombros da ciência verdadeira a responsabilidade de seguir pesquisando e compartilhando seus dados, com o objetivo de evidenciar as melhores medidas de controle, frente a pior emergência de saúde pública de interesse internacional já declarada pela OMS.

\section{REFERÊNCIAS}

1. Peiris JSM, Lai ST, Poon LLM, Guan Y, Yam LYC, Lim W, et al. Coronavirus as a possible cause of severe acute respiratory syndrome. Lancet. 2003; 361(9366):13191325.

2. Zhu N, Zhang D, Wang W, Li X, Yang B, Song J, et al. A novel coronavirus from patients with pneumonia in China, 2019. N Engl J Med. 2020; 382(8):727-733.

3. Tan W, Zhao X, Ma X, Wang W, Niu P, Xu W, et al. A Novel Coronavirus Genome Identified in a Cluster of Pneumonia Cases - Wuhan, China 2019-2020. China CDC Wkly. 2020;2(4):61-62.

4. Lu R, Zhao X, Li J, Niu P, Yang B, Wu H, et al. Genomic characterisation and epidemiology of 2019 novel coronavirus: implications for virus origins and receptor binding. Lancet. 2020; 395(10224):565-574.

5. World Health Organization (WHO). WHO Coronavirus disease (COVID-19) dashboard. [Internet]. Genebra: World Health Organization; 2021.

6. Lana RM, Coelho FC, Da Costa Gomes MF, Cruz OG, Bastos LS, Villela DAM, et al. The novel coronavirus (SARS-CoV-2) emergency and the role of timely and effective national health surveillance. Cad Saude Publica. 2020;36(3):e00019620. 
7. Organização Mundial de Saúde (OMS). Organização Pan-Americana de Saúde (OPAS). Folha informativa da COVID-19 - Escritório da OPAS e da OMS no Brasil [Internet]. Brasil: Organização Mundial de Saúde; 2021.

8. John Hapkins University (JHU). COVID-19 Dashboard by the Center for Systems Science. [Internet]. Baltimore: John Hapkins University; 2021.

9. Van Doremalen N, Bushmaker T, Morris DH, Holbrook MG, Gamble A, Williamson BN, et al. Aerosol and surface stability of SARS-CoV-2 as compared with SARSCoV-1. N Engl J Med. 2020; 382(16):1564-1567.

10. Riddell S, Goldie S, Hill A, Eagles D, Drew TW. The effect of temperature on persistence of SARS-CoV-2 on common surfaces. Virology J. 2020;17(1):1-7.

11. Prata-Barbosa A, Lima-Setta F, Sabtos GR, Lanziotti VS, Castro REV, Souza DC, et al. Pediatric patients with COVID-19 admitted to intensive care units in Brazil: a prospective multicenter study. Jornal de Pediatria. 2020; 96(5):582-592.

12. Cavalcante JB, Cardoso-dos-Santos AC, Bremm JM, Lobo AP, Macário EM, Oliveira WK, França GVA. COVID-19 en Brasil: evolución de la epidemia hasta la semana epidemiológica 20 de 2020. Epidemiol Serv Saúde. 2020; 29(4):e2020376.

13. Ministério da Saúde (BR). Boletins Epidemiológicos da COVID-19. [Internet]. Brasília: Ministério da Saúde; 2020.

14. Ministério da Saúde (BR). Guia de vigilância em epidemiológica: emergência de saúde pública de importância nacional pela doença pelo coronavírus 2019 [Internet]. Brasília: Ministério da Saúde; 2020; 58 p.

15. Wu Z, McGoogan JM. Characteristics of and important lessons from the coronavirus disease 2019 (COVID-19) outbreak in China: summary of a report of 72314 cases from the Chinese Center for Disease Control and Prevention. JAMA. 2020;323(13):1239-1242.

16. Freitas ARR, Napimoga M, Donalisio MR. Análise da gravidade da pandemia de Covid-19. Epidemiol Serv Saúde. 2020;29(2):e2020119.

17. The World Bank - IBRD - IDA. Databank: world development indicators. [Internet]. Washington, DC: The World Bank; 2020 [cited 2021 jan 2].

18. Instituto Brasileiro de Geografia e Estatística - IBGE. Perfil dos municípios brasileiros: 2017. [Internet]. Rio de Janeiro: Instituto Brasileiro de Geografia e Estatística; 2017 [citado 2021 Jan 2]. 106 p.

19. Ministério da Saúde (BR). Conselho Nacional de Saúde. Resolução $n^{\circ}$ 466, de 12 de dezembro de 2012. [Internet]. Brasília: Ministério da Saúde; 2012 [citado 2021 Jan 2].
20. Rozin L. Em tempos de COVID-19: um olhar para os estudos epidemiológicos observacionais. Rev Espaço para a Saúde. 2020; 21(1):6-15.

21. Mellan $\mathrm{T}$, Hoeltgebaum $\mathrm{HH}$, Mishra S, Whittaker $\mathrm{C}$ Schnekenberg RP, Gandy A, et al. Subnational analysis of the COVID-19 epidemic in Brazil. [Internet]. Medrxiv; 2020 august [cited 2021 Jan 2].

22. rneck GL, Carvalho MS. A pandemia de COVID-19 no Brasil: crônica de uma crise anunciada. Cad Saúde Pública. 2020;36(5):e00068820.

23. Gomes GGC, Bisco NCB, Paulo MF, Fabrin SC, Fioco EM. Perfil epidemiológico da nova doença infecciosa do coronavírus-COVID-19 (Sars-Cov-2) no mundo: Estudo descritivo, janeiro-junho de 2020. Brazilian J Health Review. 2020; 3(4):7993-8007.

24. Anderson RM, Heesterbeek H, Klinkenberg D, Hollingsworth TD. How will country-based mitigation measures influence the course of the COVID-19 epidemic? The Lancet. 2020; 395(10228):931-934.

25. Remuzzi A, Remuzzi G. COVID-19 and Italy: what next? The Lancet. 2020; 395(10231):1225-1228.

26. The Lancet. COVID-19 in Brazil: "So what?". The Lancet. 2020; 395(10235): 1461.

27. Notari A, Torrieri G. COVID-19 transmission risk factors [Internet]. Medrxiv; 2020 [cited 2021 Jan 2].

28. Guimarães RB, Catão RC, Martinuci OS, Pugliesi EA Matsumoto PSS. O raciocínio geográfico e as chaves de leitura da Covid-19 no território brasileiro. Estudos Avançados. 2020; 34(99);119-139.

29. Sabino EC, Buss LF, Carvalho MP, Prete Jr CA, Crispim MAE, Fraiji NA, et al. Resurgence of COVID-19 in Manaus, Brazil, despite high seroprevalence [Internet]. The Lancet; 2021 [cited 2021 Feb 2].

30. Palácio MAV, Takenami I. Em tempos de pandemia pela COVID-19: o desafio para a educação em saúde. Vigil Sanit Debat. 2020; 8(2):10-15.

\section{CORRESPONDÊNCIA}

Francisco Railson Bispo de Barros

Programa de Pós-Graduação em Medicina Tropical, Universidade do Estado do Amazonas,

Avenida Carvalho Leal, $n^{\circ}$ 1777- Cachoeirinha,

Manaus, Amazonas, Brasil, 69065-001

E-mail: enf.franciscobarros@gmail.com 